\title{
Mythologie der Unvernunft
}

Thomas Manns Doktor Faustus als mythenkritischer Roman

Mythologie du déraisonnable. Le Docteur Faustus de Thomas Mann et la déconstruction critique d'un mythe

Mythology of Unreason. Thomas Mann's Doctor Faustus as a Myth-Critical Novel

\section{Stefan Matuschek}

\section{OpenEdition Journals}

Édition électronique

URL : http://journals.openedition.org/ceg/4679

DOI : $10.4000 /$ ceg. 4679

ISSN : 2605-8359

\section{Éditeur}

Presses Universitaires de Provence

\section{Édition imprimée}

Date de publication : 2 mai 2019

Pagination : 163-174

ISBN : 979-10-320-0214-8

ISSN : 0751-4239

\section{Référence électronique}

Stefan Matuschek, "Mythologie der Unvernunft“, Cahiers d'Études Germaniques [Online], 76 | 2019, Online erschienen am: 02 November 2020, abgerufen am 25 Januar 2021. URL: http:// journals.openedition.org/ceg/4679; DOI: https://doi.org/10.4000/ceg.4679 


\title{
Mythologie der Unvernunft \\ Thomas Manns Doktor Faustus als mythenkritischer \\ Roman
}

\author{
Stefan MATUSCHEK \\ Universität Jena
}

Völkerpsychologische Klischees sind so irrational wie beliebt und unausrottbar. Sie können unterhaltsam sein wie in den Asterix-Heften von Goscinny und Uderzo oder unheilvoll wie in der Behauptung, der Nationalsozialismus entspräche dem deutschen Nationalcharakter. Diese Behauptung ist von beiden Seiten aufgestellt worden, von den Nationalsozialisten selbst wie von ihren Gegnern. Alfred Rosenberg und Joseph Goebbels beriefen sich für ihre Sache auf das deutsche Wesen, so wie es insbesondere in der Romantik zum Ausdruck gekommen sei, und zahlreiche Gegner der Nationalsozialisten bestätigten dies ihrerseits, indem sie schon bald nach Hitlers Amtsantritt 1933 mit Ableitungsthesen der völkischen Ideologie und Diktatur aus dem romantisch-deutschen Volksgeist aufwarteten. Wer solche Zusammenhänge herstellt, schafft einen Mythos. Denn die Vorstellung eines Nationalcharakters und die Annahme, man könne die jahrhundertelange, viele Generationen übergreifende politisch-kulturelle Entwicklung eines Landes so verstehen wie die Lebensgeschichte eines individuellen Menschen, sind erzählerische Konstruktionen. Sie können manche suggestive Analogien verwenden - wie etwa die zwischen der romantisch volkstümlichen Gemeinschaftssehnsucht und der völkischen Ideologie, erklären damit aber kaum etwas, sondern wirken vor allem als emotionale Verstehenssurrogate. Rosenberg und Goebbels dient die Berufung auf die Romantik zur eigenen kulturhistorischen Legitimierung und Verankerung, wobei sie beide ganz Gegensätzliches darunter verstehen: archaisch-chthonische Tiefgründigkeit und Gemeinschaft der eine; Seelenstärke im Angesicht modernster Technik der andere, der seine Auslegung in der Rede von der „stählernen Romantik“ der Deutschen pointierte. ${ }^{1}$ Die nationalsozialistischen Bezugnahmen auf die Romantik, so hat Ralf Klausnitzer gezeigt, sind selektiv, beliebig und widersprüchlich. Sie belegen keine historische Konsequenz von der deutschen Romantik zu Hitler. Sie erschaffen vielmehr die mythische Figur eines auserwählten oder stigmatisierten Volkes. Darin gleichen sich Rosenberg, Goebbels und deren Gegner, auch wenn sie moralisch ganz anders zu bewerten sind. Die affirmative wie die kritische Rede vom deutschen

1. Vgl. Ralf Klausnitzer, Blaue Blume unterm Hakenkreuz. Die Rezeption der deutschen literarischen Romantik im Dritten Reich, Paderborn/ München/ Wien/ Zürich, Schöningh, 1999, S. 461-489. 
Wesen, das schicksalhaft von der Romantik zum Nationalsozialismus führe, ist eine politische Emotionalisierung, die sich als Geschichtskenntnis und -diagnose ausgibt.

Auch Thomas Mann, der weltweit angesehenste und publizistisch präsenteste Gegner der Nationalsozialisten, folgt diesem Muster. Er gehört zu den ersten, die es öffentlich vertreten und verbreiten. Einen Anstoß dazu mag wohl das Buch von Helmuth Plessner gegeben haben, das 1935 unter dem Titel Das Schicksal deutschen Geistes im Ausgang seiner bürgerlichen Epoche erschien und dann nach dem Krieg von 1959 an unter dem anderen Titel Die verspätete Nation. Über die politische Verführbarkeit bürgerlichen Geistes bekannter wurde. Plessner führt den deutschen Nationalcharakter, der den Nationalsozialismus ermöglicht habe, über die Romantik hinaus bis auf Luther zurück. Thomas Mann wird diese Perspektive in mehreren Essays zwischen 1941 und 1945 und auch im Faustus-Roman aufnehmen. Plessners Buch hat er zuvor höchstwahrscheinlich gelesen. ${ }^{2}$ Plessner selbst drückt seine Solidarität mit Thomas Mann dadurch aus, dass er der Neuausgabe 1959 ein Zitat aus einem dieser Mann'schen Essays voranstellt, und zwar aus dem in diesem Zusammenhang längsten und bekanntesten: Deutschland und die Deutschen von 1945. Plessner zitiert:

Was ich Ihnen in abgerissener Kürze erzählte [...], ist die Geschichte der deutschen Innerlichkeit. Eines mag diese Geschichte uns zu Gemüte führen: daß es nicht zwei Deutschland gibt, ein böses und ein gutes, sondern nur eines, dem sein Bestes durch Teufelslist zum Bösen ausschlug [...]. Thomas Mann, Deutschland und die Deutschen $1945 .^{3}$

Die „Teufelslist“" verweist auf den Faust-Mythos und damit auf den Faustus-Roman, an dem Thomas Mann zur selben Zeit arbeitete und der seinerseits das mythische Nationalschema zur Darstellung und Reflexion der politischen Katastrophe in Deutschland heranzieht. Ein weiteres Werk, das ihn beim Roman wie bei den Essays inspiriert hat, fokussiert anders als Plessner auf die Romantik als Ursprung des deutschen Verhängnisses. Es ist eine Harvard-Dissertation von Peter Viereck, die 1941 unter dem Titel Metapolitics. From Wagner and the German Romantics to Hitler erschien. Vierecks Buch hat dazu beigetragen, dass Thomas Mann in seinen Essays von 1941 an von seinem positiven Romantik-Verständnis abrückte und stattdessen deren unheilvolle Kontinuität zum Nationalsozialismus betonte. ${ }^{4}$ Diese Sicht hat sich dann besonders durch Georg Lukács Werk Die Zerstörung der Vernunft von 1954 verbreitet, dessen späterer Untertitel so schicksalhaft teleologisch formuliert ist wie Vierecks: Der Weg des Irrationalismus von Schelling zu Hitler.

Plessner, Viereck und Lukács sind Wissenschaftler. Vierecks Buch diente sogar als akademische Qualifikationsschrift und wurde damit von einer Prüfungskommission

2. Vgl. Thomas Mann, Doktor Faustus. Das Leben des deutschen Tonsetzers Adrian Leverkühn, erzählt von einem Freunde. Kommentar von Ruprecht Wimmer unter Mitarbeit von Stephan Stachorski, Frankfurt a. M., Fischer, 2007, S. 376 (= Große kommentierte Frankfurter Ausgabe 10.2).

3. Helmuth Plessner, Die verspätete Nation. Über die politische Verführbarkeit bürgerlichen Geistes, Frankfurt a. M., Suhrkamp, 1974, S. 9.

4. Dazu mein Beitrag: „Perspektivische Amerikanisierung. Thomas Mann, Peter Viereck und die deutsche Romantik“, in Jens Ewen, Tim Lörke, Regine Zeller (Hrsg.), Im Schatten des Lindenbaums. Thomas Mann und die Romantik, Würzburg, Königshausen und Neumann, 2016, S. 197-217. 
als Nachweis wissenschaftlicher Kompetenz angenommen. Wenn man es heute liest, erscheint es einem als Verschränkung von Mythendiagnose und Mythenbildung. Das gilt auch für Plessner, Lukács und Thomas Mann. Die ideologiekritische Rekonstruktion eines verhängnisvollen deutschen Wesens ist von seiner Konstruktion ununterscheidbar. Die kritischen Diagnostiker stellen in ihren Diagnosen erst her, was sie vorzufinden meinen. Zwischen den Historikern und Germanisten auf der einen Seite und dem Schriftsteller auf der anderen besteht da kein Unterschied. Wissenschaftlich lässt sich beschreiben und analysieren, wie die Nationalsozialisten (oder auch andere zuvor) mythische Vorstellungen von der Überlegenheit, Berufung und Sendung der Deutschen geschaffen und politisch verwendet und welche alten und neuen Versatzstücke (etwa aus der Siegfried- und Nibelungensage oder der Rassenideologie) sie dazu heranzogen haben. ${ }^{5}$ Die Wissenschaftlichkeit verliert sich aber genau in dem Moment, in dem diese mythischen Vorstellungen nicht mehr als solche, sondern als tatsächliche völkerpsychologische Wesensmerkmale behandelt werden. Das Hin und Her zwischen diesen Alternativen - zwischen der rationalen Mythen-Diagnose und dem völkerpsychologischen Für-wahr-Halten dieser Mythen - zeigt sich bei Viereck am deutlichsten gegenüber Alfred Rosenbergs Mythus des 20. Jahrhunderts. Anfangs weist er darauf hin, dass Rosenberg „Mythus“ keineswegs im aufgeklärt distanzierten Sinne verstehe, sondern ganz im Gegenteil als ,notwendigen Glauben [...], wahrer als Wahrheit". ${ }^{6}$ Für Viereck selbst und seine Leser heißt das, die Distanz zu wahren und Rosenbergs Werk als ,Mythos' im aufgeklärt skeptischen Sinne zu nehmen und damit als Propaganda-Konstruktion zu durchschauen. In der Art jedoch, wie Viereck sich dieses Werk dann vornimmt, verliert sich die Distanz immer wieder in der stillschweigenden Annahme, dass sich darin eben doch das wahre Wesen der Deutschen zeige. So verfällt Viereck dem völkischen Mythos, den er kritisch analysieren will. Dasselbe Hin und Her zwischen Mythendiagnose und Mythenbildung zeigt auch Thomas Mann, wenn er die nationalsozialistische Ideologie einerseits als „mythisches Surrogat" und ausdrücklich auch als „Lüge“7 bezeichnet und sie andererseits im Sinne einer aufrichtigen ,deutschen Selbstkritik“" als Ausdruck des eigenen Nationalcharakters bestätigt.

Die Schnelligkeit und Intensität, in der die nationalsozialistische Gleichschaltung von 1933 an gelang, sowie dann das Ausmaß der nationalsozialistischen Verbrechen lassen verstehen, warum die ersten kritischen Diagnosen so emotional und mythenaffin waren. Was zwischen 1933 und 1945 in Deutschland und den von Deutschland besetzten Gebieten geschah, war so ungeheuerlich, dass die politische und soziologische Urteilskraft überfordert schienen. Es hatte offenbar eine viel

5. Z. B. Herfried Münkler, Die Deutschen und ihre Mythen, Berlin, Rowohlt, 2009.

6. „necessary faith [...], truer than truth“. Peter Viereck, Metapolitics. From Wagner and the German Romantics to Hitler. Expanded edition. With a new introduction by the author, New Brunswick/ London, Transaction Publishers, 2007, S. 229.

7. Mann, Richard Wagner und der ,Ring des Nibelungen ' [1937], in T. M., Leiden und Größe der Meister, hrsg. und mit Nachbemerkungen versehen von Peter de Mendelssohn, Frankfurt a. M., Fischer, 1982, S. 779-804, hier S. 803.

8. Mann, Deutschland und die Deutschen [1945], in Th. M., An die gesittete Welt. Politische Schriften und Reden im Exil. Nachwort von Hanno Helbling, hrsg. von Peter de Mendelssohn, Frankfurt a. M., Fischer, 1986, S. 701-723, hier S. 721. 
größere Dimension, als mit den wenigen Jahren diktatorischen Regierungshandelns zu erklären war. Genau diese Überzeugung vereint Plessner, Viereck, Thomas Mann und Lukács. Sie alle sehen sich gezwungen, historisch weit auszuholen, um der Dimension der NS-Ideologie und -Diktatur gerecht zu werden. Der Triumph, die Verbrechen und das katastrophale Ende der NSDAP waren zu gewaltig, als dass sie die deutsche Geschichte dabei anders als in diesem Lichte sehen konnten. Auch das vereint Plessner, Viereck, Mann und Lukács. Im Angesicht des noch zeitgenössischen oder gerade überwundenen Nationalsozialismus musste ihnen die deutsche Geschichte unweigerlich als dessen Vorgeschichte erscheinen. Vorgeschichten aber sind, wenn sie etwas erklären sollen, teleologisch. Und eine Teleologie, die fünf oder auch nur zwei Jahrhunderte deutscher Geschichte auf die Linie einer individual- oder kollektivpsychologischen Abnormitätsentwicklung bringt, ist nicht anders als mythisch zu nennen. Es ist jedoch aus ihrer Situation heraus verständlich, warum Plessner, Viereck, Mann und Lukács so verfahren. Mit ihren historischvölkerpsychologischen Deutungen versuchen sie, den schockierenden deutschen Maßlosigkeiten mit einem ersten menschlichen Erklärungsmaß zu begegnen. Und Mythen kann man insofern als ,menschliche“ Erklärungen bezeichnen, als sie das affektive und emotionale Verhältnis zur erklärten Sache mit zum Ausdruck bringen. Mehr als die drei Wissenschaftler macht Thomas Mann diese Dimension seiner völkerpsychologischen Deutung explizit:

Nichts von dem, was ich Ihnen über Deutschland zu sagen oder flüchtig anzudeuten versuchte, kam aus fremdem, kühlem, unbeteiligtem Wissen; ich habe es auch in mir, ich habe alles am eigenen Leibe erfahren. ${ }^{9}$

Der Faustus-Roman folgt der in den Essays eingenommenen Perspektive. Deren bekanntester, Deutschland und die Deutschen, hält die Grundidee des Werks fest, wenn er die politische Situation Deutschlands 1945 mit der Faust-Sage vergleicht, und zwar mit dem tragischen Ausgang, wie ihn das Volksbuch erzählt. Wie dieser alte Faust werde Deutschland nun „,buchstäblich“ vom Teufel geholt; von Luthers und Faustens Teufel, der Thomas Mann ,als eine sehr deutsche Figur“ erscheint. Es sei nur ein Fehler der Sage, dass sie Faust nicht als einen Musiker darstelle. Denn mit dieser Kunst lasse sich die dämonische Tiefe des deutschen Wesens am besten erfassen. ${ }^{10}$ Der Roman verfährt genau so. Er konstruiert die Biographie eines deutschen Komponisten, die mit vielen Motiven (insbesondere dem Teufelspakt) aus der Faustsage, aber auch zahlreichen weiteren deutschen Topoi ausgestattet ist. Das beginnt schon mit dem Untertitel, der Adrian Leverkühn nicht als Komponisten, sondern altertümlich nationalsprachlich als „deutschen Tonsetzer“ bezeichnet. Sein Geburtsort „Kaisersaschern“, im mitteldeutschen Kernland des Protestantismus angesiedelt, wird als Inbegriff gesellschaftlicher Antimoderne mit latenter Rückschlagsgefahr in abergläubische Barbarei gezeichnet. Der Hinweis auf „Bücherverbrennungen“ $(58)^{11}$ schlägt dabei einen unverkennbaren

9. Ibid.

10. Vgl. Ibid, S. $705 f$.

11. Alle Zitate aus dem Roman werden durch Angabe der Seitenzahl nach folgender Ausgabe nachgewiesen: Thomas Mann, Doktor Faustus. Das Leben des deutschen Tonsetzers Adrian 
Bogen zum Nationalsozialismus. Leverkühns Studienzeit bringt deutschnationale Burschenschaftlergespräche, seinTheologiestudiuminHallemitdemProfessorKumpf trutziges Lutherdeutsch und mit dem Dozenten Schleppfuß Vorausdeutungen auf den Teufel. Schon die Namen (Kumpf, Schleppfuß) klingen klischeehaft schwerfällig konsonantisch deutsch. Dass Leverkühn sein Musikstudium als konsequente Fortsetzung, ja Überbietung der Theologie mit künstlerischen Mitteln versteht, bedient das Tiefsinnsklischee der deutschen Musik. Seine Syphilis-Infektion stiftet eine Analogie zu Nietzsches Biographie und erlaubt eine moderne Rationalisierung der Teufelsverschreibung als Psychopathologie. Seine Werkgeschichte reflektiert die politische Entwicklung Deutschlands von der Romantik bis zum Zweiten Weltkrieg. Seine letzte Komposition, die Vertonung von „D. Fausti Weheklag“ (709), nach einer Kapitelüberschrift des Volksbuchs Historia von D. Johann Fausten von 1587, stellt mit dem tragischen Ende der frühneuzeitlichen Faust-Sage allegorisch den Zustand Deutschlands nach dem Kriegsende dar. Im Schlusssatz des Romans könnte man in der Stimme des fiktiven Erzählers Zeitblom zugleich die des realen Autors Thomas Mann hören: „Ein einsamer Mann faltet seine Hände und spricht: Gott sei euerer armen Seele gnädig, mein Freund, mein Vaterland.“" (738)

Dass Thomas Mann die Faust-Sage für seinen Deutschland-Roman wählt, liegt nahe. Zusammen mit der Siegfried-Figur ist Faust seit der Reichsgründung 1870/71 intensiv popularisiert und mythenbildend in den Dienst des deutschen Nationalstolzes gestellt worden. Das Prestige von Goethes Drama als Gipfelwerk der deutschen Nationalliteratur eröffnete dabei die Spannweite vom akademischen bis zum Populär-Diskurs. Zugleich bietet diese Goethe-Rezeption den wohl krassesten Beleg, wie sich die ideologische Verwendung eines literarischen Werkes verselbstständigen und dessen Intention geradezu ins Gegenteil kehren kann. Denn populär und für den deutschen Nationalstolz wirksam geworden ist Goethes Faust durch die Ideologie des ,faustischen Menschen'. Anders als das Drama selbst versteht sie den Helden als positive Identifikationsfigur: als ewig strebsamen Erkenntnissucher und Tatmenschen, der trotz der Opfer, die seinen Lebensweg säumen, eben aufgrund seiner unermüdlichen Strebsamkeit gerechtfertigt sei. In dieser Deutung wurde Goethes Faust zum Prototyp des deutschen Wesens erhoben. Goethes Text zeigt seinen Helden dagegen kritisch und mit distanzierter Ironie. ${ }^{12}$

Manns Doktor Faustus führt die Figur an Goethes Fassung vorbei auf das alte, fromme Volksbuch von 1587 zurück. Anders als bei Goethe fährt der Teufelsbündler hier zur Hölle. Der Roman entwickelt daraus die große Perspektive, dass alle Zuversicht in der deutschen Geschichte (symbolisch in Fausts Himmelfahrt in Goethes Dramenschluss ausgedrückt) trügerisch sei, die Katastrophe dagegen von Beginn an eingeschrieben und unvermeidlich, definitiv. Die in der Überlieferung erste und Thomas Manns letzte Fassung der Faust-Sage entsprechen sich in ihrem

Leverkühn, erzählt von einem Freunde. Hrsg. und textkritisch durchgesehen von Ruprecht Wimmer unter Mitarbeit von Stephan Stachorski, Frankfurt a. M., Fischer, 2007 (= Große kommentierte Frankfurter Ausgabe 10.1)

12. Vgl. dazu meinen Beitrag „Pathostransfer. Wagners Beitrag zum Faust-Mythos“, in Stephanie Wodianka, Juliane Ebert (Hrsg.), Inflation der Mythen? Zur Vernetzung und Stabilität eines modernen Phänomens, Bielefeld, Transcript, 2016, S. 195-218. 
bösen Ende; Goethes Rettungsvariante wird als falsche Hoffnung korrigiert. Die Kompositionsbeschreibung der „Weheklag“ sagt dasselbe im Blick auf die Musik. Leverkühns düsteres und trostloses Werk erscheint als „Zurücknahme“ der 9. Beethoven-Symphonie und ihrer Freuden-Ode: „[...] es ist gleichsam der umgekehrte Weg des ,Liedes an die Freude“, das kongeniale Negativ [...], es ist die Zurücknahme...“ (709). Eine minimale Hoffnungsperspektive bleibt als Paradox und in Frageform: „daß aus tiefster Heillosigkeit, wenn auch als leiseste Frage nur, die Hoffnung keimte?“ (711) In der Musikbeschreibung steht dafür symbolisch ,das hohe g eines Cello [...], in pianissimo-Fermate langsam vergehend“ (711). Man liegt nicht falsch, wenn man in diesem paradoxen Hoffnungsrest den Einfluss von Theodor W. Adorno erkennt. In den zur selben Zeit entstandenen Minima Moralia formuliert Adorno ganz analog, dass ,die vollendete Negativität, einmal ganz ins Auge gefaßt, zur Spiegelschrift ihres Gegenteils zusammenschießt" und sich so aus der aktuellen „Verzweiflung“ indirekt der „Standpunkt der Erlösung“ und dessen „Messianisches Licht“" ergeben. ${ }^{13}$ In der Entstehung des Doktor Faustus hat Thomas Mann mitgeteilt, wie sehr Adorno bei der Beschreibung der Weheklag-Komposition die Feder führte, und zwar ausdrücklich auch ,auf dem Gebiet der Sprache und ihrer Nuancen“, die, „ganz zuletzt, ein Moralisches, Religiöses, Theologisches umwerben". ${ }^{14}$

Schaut man auf diese faustische, um viele weitere deutsche Klischees angereicherte Komponisten-Biographie, wird verständlich, warum man dem Faustus-Roman vorgeworfen hat, er trage seinerseits zur Mythisierung und damit zur irrationalen Behandlung der deutschen Geschichte bei. Mit größter Klarheit hat Peter Michelsen diesen Vorwurf erhoben: Manns Roman „reaktiviere“ den „diabolischen Kern“ der Faust-Sage, um sie gegen das Ideologem des Faustischen und dessen vom Wilhelminismus bis zum Nationalsozialismus reichende „Hybris tragischer Erwähltheit" zu wenden; womit allerdings nichts erhellt, sondern nur das Vorzeichen umgekehrt und der nationalistische als anti-nationalistischer Mythos fortgeschrieben werde. ${ }^{15}$ Wäre der Roman nichts anderes als die KomponistenBiographie und wäre der Autor Thomas Mann ihr Erzähler, bestünde dieser Vorwurf zu Recht.

Doch ist der Roman mehr und ist der Autor nicht der Erzähler. Denn der Roman stellt zusammen mit der Biographie auch deren Erzählvorgang dar. Das hebt schon der Untertitel hervor: „Das Leben des deutschen Tonsetzers Adrian Leverkühn, erzählt von einem Freunde.“ Nun ist es ein gängiges Kunstmittel, dass Autoren Erzählerfiguren konstruieren und mit ihnen Erzählsituation und -perspektive

13. Theodor W. Adorno, Minima Moralia. Reflexionen aus dem beschädigten Leben, 21. Aufl., Frankfurt a. M., Suhrkamp, 1993, S. 333f.

14. Mann, Die Entstehung des Doktor Faustus, in: Essays VI, 1945-1950. Hrsg. und textkritisch durchgesehen von Herbert Lehnert, Frankfurt a. M., Fischer, 2009, S. 409-581, hier S. 573 (= Große kommentierte Frankfurter Ausgabe 19.1).

15. Vgl. Peter Michelsen, „Faust und die Deutschen (mit besonderem Hinblick auf Thomas Manns Doktor Faustus)", in Peter Boerner, Sidney Johnson (Hrsg.), Faust through Four Centuries. Retrospect and Analysis. Vierhundert Jahre Faust. Rückblick und Analyse, Tübingen, Max Niemeyer Verlag, 1989, S. 229-247, hier S. 246 f. 
ausdrücklich als Teil der Erzählung mitgestalten. Thomas Mann gibt dem hier ein besonderes Gewicht und macht den Erzähler zur zweiten, kontrastiven Hauptfigur. Serenus Zeitblom und Adrian Leverkühn stehen sich als Bürger und Künstler, als braver Beamter und dämonisches Genie, als gesellschaftlicher Durchschnitt und als auratischer Einzelner gegenüber. Thomas Mann selbst hat dies zum entscheidenden Kunstgriff erklärt, durch den der Roman erst möglich geworden sei. ${ }^{16}$ Der Kontrast der beiden Figuren - vor allem in der umständlichen Art, wie er von Zeitblom ausdrücklich bedacht und zelebriert wird, gewinnt dem schweren, düsteren Stoff etwas Komisches hinzu und heitert ihn zur Romanunterhaltung auf. Auch schafft die Tatsache, dass nur Zeitblom und nicht Leverkühn in der Nazi- und Kriegszeit platziert ist, eine Distanz zwischen dem Faust-Wiedergänger und dem Nationalsozialismus. Sie ist wichtig, um die mythische Deutungsfigur nicht zu plump auf die deutsche Politik zu beziehen. Als unpolitischer, einzelgängerischer, in intellektueller Askese zurückgezogener Komponist, der zu Beginn der 1930er Jahre in psychischer Krankheit versinkt und dann 1940 stirbt, bleibt Leverkühn auf der Handlungsebene konsequent vom Nationalsozialismus getrennt. Ein Zusammenhang ergibt sich nur aus Zeitbloms Perspektive, der die Kompositionen seines Freundes so beschreibt und interpretiert, dass sie als Allegorien auf die gesellschaftlich-mentale Entwicklung in Deutschland von der Jahrhundertwende über den Triumph der Nationalsozialisten bis zum Untergang im Krieg erscheinen.

Es ist also, genau genommen, nicht der Roman, sondern seine Erzählerfigur, die den Bezug zwischen Leverkühn und der deutschen Politik herstellt. Das ist ein entscheidender Unterschied. Denn das mythische Deutschlandbild, das so entsteht, rückt damit auf Distanz und wird als Deutung und Sichtweise einer bestimmten Figur analysierbar. Nicht der Roman mythisiert die deutsche Geschichte, er entwirft vielmehr einen Erzähler, der das tut. Zeitblom wird zum Anschauungsbeispiel, dass und wie jemand die deutsche Geschichte zum Mythos macht. Nicht der Autor Thomas Mann, sondern die von ihm konstruierte Figur Serenus Zeitblom spricht, wie sie bekennt, „mit einer gewissen Ergriffenheit“ von dem „mythischen Hervortreten der National-Charaktere“ (443). In der Romanhandlung steht dieses Bekenntnis im Zusammenhang mit dem Ersten Weltkrieg, so dass man es, denkt man nur an Manns Betrachtungen eines Unpolitischen von 1918, tatsächlich auch auf den Autor beziehen kann. Dann aber erscheint die Zeitblom-Figur als ein Ausdruck von Thomas Manns Selbstkritik und Distanzierung. Denn so humanistisch friedfertig und betulich harmlos sich dieser durch seinen Vornamen als heiter und ruhig (serenus) ausgewiesene Gelehrte auch gibt, so steht er am Ende doch als ein von seiner ,mythischen Ergriffenheit' beschädigtes Bewusstsein da. Zeitblom ist die Figur, in der die mythisierende Deutschland-Deutung als Psychopathologie erkennbar wird. Darin liegt die besondere Leistung des Doktor Faustus-Romans.

Der Erzähler erscheint in seiner eigenen Gesinnung ambivalent. Er verkörpert den passiven Widerstand gegen den Nationalsozialismus, die ,Innere Emigration “ und man darf wohl sagen, dass der Emigrant Thomas Mann aus dem amerikanischen Exil heraus diese Haltung mit Zeitblom sehr kritisch, anklagend darstellt. Zwar ist

16. Vgl. Mann, Entstehung (wie Anm. 14), S. 429f. 
Zeitblom so konsequent, dass er aus politischen Gründen seinen Lehrerberuf opfert. Doch formuliert er diesen Standpunkt so, dass er den Nationalsozialismus dabei beschönigt, sogar legitimiert und sich nur herauszuhalten versucht:

[Meiner] Gesinnung habe ich Opfer gebracht, ideelle und solche des äußeren Wohlseins, indem ich ohne Zögern meinen mir lieben Lehr-Beruf vor der Zeit aufgab, als sich erwies, daß sie sich mit dem Geiste und den Ansprüchen unserer geschichtlichen Entwicklungen nicht vereinbaren ließ. In dieser Beziehung bin ich mit mir zufrieden. (12)

Wer die nationalsozialistische Machtergreifung als ,Geist und Anspruch unserer geschichtlichen Entwicklungen` bezeichnet, hat sich von der völkischen Ideologie kaum befreit und hält sich nur, wie der letzte Satz zeigt, selbstgerecht auf gewissem Abstand. Daraus resultiert ein Patriotismus, in dem sich Erschrecken und Faszination über ein mythisches Deutschland-Bild auf groteske Weise verbinden. Erschrocken ist Zeitblom im Rückblick über seine nationalistische Kriegsbegeisterung 1914. Als ein „Riesenrausch“ kommt sie ihm nun vor und schärfer noch: als ein „Giftfusel“ und „ordinär schwelgerischer Kult eines Hintertreppenmythos“ (256). Erschrocken ist er auch über die aktuelle Verschärfung und politische Mobilmachung dieses Deutschland-Mythos, wie er sie im Münchner Kridwiß-Kreis erlebt. Mit ihm stellt der Roman das großbürgerliche und intellektuelle Milieu dar, in dessen Resonanzraum sich die völkische Ideologie entwickelt und die NSDAP ihre ,Hauptstadt' gefunden hat. Durch die Lektüre von Sorels Réflexions sur la violence herrscht in diesem Kreis ein begeistertes wie zynisches Bewusstsein von der Massenwirksamkeit des Mythos. Zeitblom erlebt und referiert dies mit Schrecken:

Dieses war in der Tat die krasse und erregende Prophetie des Buches, daß populäre oder vielmehr massengerechte Mythen fortan das Vehikel der politischen Bewegung sein würden: Fabeln, Wahnbilder, Hirngespinste, die mit Wahrheit, Vernunft, Wissenschaft überhaupt nichts zu tun zu haben brauchten, um dennoch schöpferisch zu sein, Leben und Geschichte zu bestimmen und sich damit als dynamische Realitäten zu erweisen. [...] Sie machten sich den Spaß, eine Gerichtsverhandlung zu imaginieren, in welcher einer jener dem politischen Antrieb, der Unterwühlung der bürgerlichen Gesellschaftsordnung dienenden Massenmythen zur Diskussion stand, ihre Protagonisten sich gegen den Vorwurf der „Lüge“ und „Fälschung“ zu verteidigen hatten [...]. Das Groteske war der gewaltige Apparat wissenschaftlicher Zeugenschaft, den man aufgeboten hatte, um den Humbug als Humbug, als skandalösen Affront gegen die Wahrheit zu erweisen, da doch der dynamisch-geschichtsschöpferischen Fiktion, der sogenannten Fälschung, das heißt: dem gemeinschaftsbildenden Glauben von dieser Seite gar nicht beizukommen war und ihre Verfechter desto höhnisch-überlegenere Gesichter machten, je emsiger man sich mühte, sie auf ganz fremder und für sie irrelevanter Ebene, der wissenschaftlichen nämlich, der Ebene der biederen, objektiven Wahrheit zu widerlegen. (532 f. $)^{17}$

17. Aus der Defensive heraus wird das hier Beschriebene durch das Zeugnis von Wilhelm Böhm bestätigt, der als gewissenhafter Goethe-Philologe gegen dessen Vereinnahmung für die Ideologie des ,Faustischen' die Stimme erhob. Obwohl sein Buchtitel Faust der Nichtfaustische es an Deutlichkeit nicht fehlen lässt, respektiert Böhm doch die politische Realität der Goethefälschung: „Ich verkenne gar nicht, daß das Wort vom ,faustischen Menschen“ in neuerer Zeit durchaus eine Kulturmission hat, und Missionen müssen durch Legenden wirken!“ (Wilhelm Böhm, Faust der Nichtfaustische, Halle, Niemeyer, 1933, S. 2). Überhaupt kann die faustische Goethe-Interpretation als Musterfall für den hier beschriebenen ,massengerechten Mythos` gelten, gegen den jeder wissenschaftliche Einwand hilflos ist. 
Eine ebenso ausführliche wie prägnante Diagnose der durch Aufklärung unangreifbaren Macht politisch mobilisierender Mythen. Zeitblom erkennt diese Macht. Und zum Beweis, dass sie sich auch gegen seine Aufklärung behauptet, bleibt er ihr verfallen. Denn trotz der Scham über seine mythentrunkene Weltkriegsbegeisterung und trotz der mythenkritischen Diagnose im Kridwiß-Kreis kann er sich von seinem mythischen Deutschlandbild nicht lösen. Er ist es (und nicht Thomas Mann), der den nationalistischen als anti-nationalistischen DeutschlandMythos fortschreibt. Sein Erschrecken über die mobilmachenden Mythen führt nicht zur Abwendung von ihnen, sondern zur düsteren Faszination, in der er sich mit zynisch-bitterem, selbstanklagend dämonisierendem Patriotismus den deutschen Klischeebildern und Schicksalserzählungen hingibt. Die alten Muster bleiben, sie werden nur höhnisch pervertiert. Das ist der für Zeitblom signifikante Ton. So spricht er von der Gewissenhaftigkeit und dem Anstand der Deutschen, wo es tatsächlich um ein heimtückisches barbarisches Rückzugsmanöver geht (in der Mitteilung, dass die deutschen Truppen die Stadt Neapel „,nach gewissenhafter Zerstörung der Bibliothek und mit Hinterlassung einer Zeitbombe im Hauptpostamt, erhobenen Hauptes geräumt haben“, 255), spricht er angesichts des vernichtenden Krieges von den „,deutschen Werde-Prozessen (und wir werden ja immer)“ (438). Den Gipfel erreicht sein verzweifelt perverser Patriotismus dort, wo er sich ausmalt, dass seine dem Nationalsozialismus anhängenden Söhne ihn bei der Gestapo anzeigen müssten, wenn sie von seinem Manuskript erführen. Die „Abgründigkeit des Konfliktes“, in den seine Söhne dadurch zwischen Vater und Staat gerieten, empfindet Zeitblom, wie er sagt, „mit einer Art patriotischem Stolz“ (51). Die „Hybris tragischer Erwähltheit“18, die Michelsen dem Faustus-Roman vorwirft, trifft nicht den Roman, sondern nur dessen Erzählerfigur. Zeitblom bekennt sie wörtlich: „[...] ich bin nicht frei von der Neigung, für dieses Schicksal eine besondere, nie dagewesene Tragik in Anspruch zu nehmen [...]“ (51). Dass der Roman dieselbe Neigung zeigte oder dass er sie auch nur nahelegte oder anböte, kann man nicht sagen. Er verurteilt sie vielmehr, indem er sie hier auf den perversen Stolz zuspitzt, den ein Vater darüber empfindet, dass seine eigenen Söhne zu seinen Feinden und Verrätern werden müssen. Der Grenzwert, den dieser Patriotismus erreicht, ist nach Zeitbloms eigenem Bekenntnis „die Verzweiflung, ist der Wahnsinn“ (255). Zeitblom nimmt sie als eine Art Strafe an, wobei er das zweite Wort, „Wahnsinn“, gerade durch ein stilistisch grandioses ausdrückliches Verschweigen als angstbesetzte Selbstdiagnose pathetisch steigert: Es „muß bezahlt werden? Womit? Ich habe das Wort schon genannt, in Verbindung mit dem Wort, Verzweiflung' sprach ich es aus. Ich werde es nicht wiederholen. Nicht zweimal überwindet man das Grauen“ (256). Kurzum: Zeitblom erscheint als eine durch ihren Patriotismus psychisch zerstörte Figur. Dass er sich dennoch nach Kriegsende, so als wäre nichts gewesen, gleich wieder für den Schuldienst tauglich hält ${ }^{19}$, kann man als bitteren Kommentar zur Nachkriegssituation in Deutschland

18. Michelsen (wie Anm. 15).

19. ,[...] es mag sein, daß meiner Rückkehr in den Schuldienst bald nichts mehr im Wege stehen wird. Monsignore Hinterpförtner hat mich schon gelegentlich darauf hingewiesen." (731) Der sprechende Name bewertet seinerseits diese Rückkehr in die Normalität. 
lesen. Darin zeigt sich abermals das scharfe Urteil des Exilanten Mann über die inneren Emigranten.

In der Entstehung des Doktor Faustus spricht Thomas Mann von der „Gefahr, mit [s]einem Roman einen neuen deutschen Mythos kreieren zu helfen, den Deutschen mit ihrer ,Dämonie“ zu schmeicheln“. ${ }^{20}$ Die kritische Distanz, auf die der Roman seinen Mythenbildner Zeitblom rückt, ist ein Mittel, diese Gefahr zu bannen.

Blickt man von hier aus zurück auf Thomas Manns Essays der Jahre 1941-45, erscheint der Autor indes selbst in genau der Rolle, die er seinem Romanerzähler in kritischer Absicht zuschreibt. Wenn Zeitblom die nationalsozialistische „Gesinnung“ schon ,in den Zügen unserer Großen, der an Figur gewaltigsten Verkörperungen des Deutschtums ausgeprägt findet“" (698), dann entspricht er ganz und gar den Mann'schen Vorträgen zu diesem Thema und er trifft auch deren Ton, wenn er diese Verbindung ,nicht ohne Scheu“ (698) herstellt und hinzufügt, dass eine „Vaterlandliebe“, die diesen Zusammenhang leugnen wollte, ihm „hochherziger, als [...] gewissenhaft dünkte“ (698). Es ist der Ton einer leidvollen, bekenntnishaft abgerungenen Selbstkritik, die auf die eigene Verbindung zu dem spekulativ entworfenen Nationalcharakter reflektiert. Schon 1939 hat Thomas Mann diese Perspektive mit seinem provokanten Essay-Titel Bruder Hitler ${ }^{21}$ eröffnet. In Deutschland und die Deutschen spricht er von „Selbstprüfung“22 und rückt seinen Essay damit ins Licht persönlich-moralischer Introspektion. Angesichts der Weite des im Essay verhandelten Themas - die deutsche Geschichte und der in die Katastrophe führende deutsche Nationalcharakter - evoziert dies eine ganz unpassende intime Enge. Thomas Mann spitzt diesen Kontrast in dem Bekenntnis zu: „[...] ich habe alles am eigenen Leibe erfahren“. ${ }^{23}$ Bedenkt man, dass der Text von der deutschen Geschichte seit Luther und von den gerade zurückliegenden Kriegsgräueln handelt, ist das eine starke, geradezu groteske Emotionalisierung. Sie erklärt sich aus der Disproportion, schon kurz nach der deutschen Kapitulation mit einem individuellen, fast spontanen Text erklären zu wollen, wie es zu all dem hat kommen können. Die mythische Völkerpsychologie bietet sich als propagandistisch etabliertes Muster an, das man nur in der moralischen Bewertung zu invertieren braucht, um sich der Dimension der Sache mit der eigenen Deutung gewachsen zu zeigen. Doch werden damit vor allem die affektischen und kaum die intellektuellen Erklärungsbedürfnisse gestillt. In der Romanfigur Zeitblom wird dieser Inversionsmechanismus, dem der Redner und Essayist Thomas Mann selbst unterliegt, literarisch vergegenwärtigt und damit der kritischen Reflexion zugänglich. Die künstlerische Form wirkt hier erkenntnisfördernd. Indem Thomas Mann seine eigene Rolle des mythisierenden Deutschlanderklärers als Romanfigur distanziert und stilisiert, wird etwas sichtbar, was sich in den expliziten Überlegungen des Redners und Essayisten nicht findet: dass propagandistische Mythen die Urteilskraft nicht nur der Mitläufer, sondern auch der Gegner und Kritiker beeinträchtigen können.

20. Mann, Entstehung (wie Anm. 14), S. 448.

21. In Mann, An die gesittete Welt (wie Anm. 8), S. 253-260.

22. Mann, Deutschland und die Deutschen (wie Anm. 8), S. 703.

23. Ibid., S. 721. 
Thomas Manns Doktor Faustus ist damit einer der ergiebigsten Romane, um sich die psychologische Macht politischer Mythen zu vergegenwärtigen und bewusst zu machen. Er zeigt sie in der historischen Entwicklung in Deutschland vom Ersten zum Zweiten Weltkrieg und er zeigt - das ist seine eigene, besondere Pointe -, wie jemand dieser Macht unterliegt, der diese Mythen ideologisch ablehnt und kritisch analysiert. Mythen können wirksam bleiben, auch wenn man sie als solche erkennt. Denn sie sind Ausdruck eines emotional-affektischen Welt- und Selbstbildes, in das man sich hineinreden und in dem man gefangen bleiben kann, auch wenn man es mythenkritisch als Fiktion entlarvt. Genau das führt der Roman an der mythischen Völkerpsychologie der Deutschen vor, in der propagandistischen Mobilmachung wie in deren katastrophalem Ergebnis. Die durchschlagende Macht des Mythischen erweist sich dabei darin, dass es als Deutungsschema in der Katastrophe nicht untergeht, sondern ideologiekritisch-moralisch invertiert fortbesteht. Insofern er dies an der Zeitblom-Figur sichtbar macht, ist der Romanautor einen Erkenntnisschritt weiter als der Essayist Thomas Mann; nicht theoretisch explizit, doch mit der Vergegenwärtigungsleistung der literarischen Form. Der Faustus-Roman ist damit kein Fall von, sondern ein Lehrstück über deutschnationale Mythisierung. Er zeigt keinen mythischen Volkscharakter, sondern den Prozess, wie man ihn herbeiredet. 
